$11-1-2016$

\title{
The Application of Legendre Multiwavelet Functions in Image Compression
}

Elham Hashemizadeh

Department of Mathematics, Karaj Branch, Islamic Azad University, Karaj, Iran, hashemizadeh@kiau.ac.ir

Sohrab Rahbar

Islamic Azad University, Karaj, Iran, rahbar@irost.org

Follow this and additional works at: http://digitalcommons.wayne.edu/jmasm

Part of the Applied Statistics Commons, Social and Behavioral Sciences Commons, and the Statistical Theory Commons

\section{Recommended Citation}

Hashemizadeh, Elham and Rahbar, Sohrab (2016) "The Application of Legendre Multiwavelet Functions in Image Compression," Journal of Modern Applied Statistical Methods: Vol. 15 : Iss. 2 , Article 31.

DOI: $10.22237 /$ jmasm/1478003340

Available at: http://digitalcommons.wayne.edu/jmasm/vol15/iss2/31

This Regular Article is brought to you for free and open access by the Open Access Journals at DigitalCommons@WayneState. It has been accepted for inclusion in Journal of Modern Applied Statistical Methods by an authorized editor of DigitalCommons@WayneState. 


\section{The Application of Legendre Multiwavelet Functions in Image Compression}

\author{
Elham Hashemizadeh \\ Islamic Azad University, \\ Karaj, Iran
}

\author{
Sohrab Rahbar \\ Islamic Azad University \\ Karaj, Iran
}

Legendre multiwavelets are introduced. These functions can be designed in such a way that the properties of orthogonality, polynomial approximation, and symmetry hold at the same time. In this way, they can be effectively deployed in image compression.

Keywords: Image compression, Legendre multiwavelet, multiwavelets, preprocessing, wavelets

\section{Introduction}

In recent years, the wavelets theory has played a significant role in signal processing, especially in image processing. These wavelets are mainly scalar, where there is only one scaling function. However, multiwavelets are based on more than one scaling function. Such growing interests in multiwavelets mainly stem from the following facts: (i) multiwavelets can simultaneously possess orthogonality, symmetry, and a high order of approximation for a given support of the scaling functions (this is not possible for any real valued scaler wavelets); and (ii) multiwavelets have produced promising results in the areas of image compression.

A multiwavelet system can provide perfect reconstruction while preserving length (orthogonality), good performance at the boundaries (via linear-phase symmetry), and a high order of approximation (vanishing moments). Thus, multiwavelets offer the possibility of superior performance for image processing applications, compared with scalar wavelets. In this paper, we use linear Legendre multiwavelets in image compression, and show its usefulness through actual examples.

Dr. Hashemizadeh is an Assistant Professor in the Department of Mathematics. Email her at: hashemizadeh@kiau.ac.ir. Dr.Rahbar is a Professor in the Department of Mathematics. 


\section{HASHEMIZADEH \& RAHBAR}

\section{Multiwavelets}

As with scalar wavelets, multiwavelets are based on the concept of multiresolution analysis (MRA). The only difference is the number of scaling functions used to generate those functions. The standard multiresolution has one scaling function $\phi(t)$, which satisfies the following properties (Aboufadel \& Schilicker, 1999; Martin \& Bell, 2001, Burrus \& Gopinath, 1998; Daubechies, 1992).

- $\quad$ The translates $\phi(t-k)$ are linearly independent and produce a basis for the subspace $\phi_{1}(t), \ldots, \phi_{N}(t)$.

- $\quad$ The dilates $\phi\left(2^{j} t-k\right)$ generate subspaces $V_{j}, j \in Z$, such that $\ldots \subset V_{-1} \subset V_{0} \subset V_{1} \subset \ldots \subset V_{j} \subset \ldots \overline{\cup_{j=-\infty}^{\infty} V_{j}}=L^{2}(R), \cap_{j=-\infty}^{\infty} V_{j}=\{0\}$.

- To obtain the subspace $V_{1}$, it is sufficient to add the family of functions $\psi(t-k), W_{0}$, to $V_{0}$, i.e., $V_{1}=V_{0} \oplus W_{0}$.

In multiwavelets, the notion of MRA is used in the same way except that the basis for $V_{0}$ is generated by the translates of $N$ different scaling functions $\phi_{1}(t-k)$, $\phi_{2}(t-k), \ldots, \phi_{N}(t-k)$. As in the scalar case, the vector $\Phi(t)=\left[\phi_{1}(t), \ldots, \phi_{N}(t)\right]^{T}$, satisfies the matrix dilation equation

$$
\Phi(t)=\sum_{k} C[k] \Phi(2 t-k)
$$

where $C[k]$ is an $N$ by $N$ matrix of coefficients. There are also $N$ wavelets $\psi_{1}(t), \ldots, \psi_{N}(t)$ satisfying the matrix wavelet equation

$$
\Psi(t)=\sum_{k} D[k] \Phi(2 t-k)
$$

where $\Psi(t)=\left[\psi_{1}(t), \ldots, \psi_{N}(t)\right]^{T}$ is a vector, and $D[k]$ is an $N$ by $N$ matrix.

The scaling functions $\phi_{1}(t), \ldots, \phi_{N}(t)$ are in $V_{0}$ whose basis is $\left\{\sqrt{2} \phi_{i}(2 t-k)\right.$ : $1 \leq i \leq N, k \in Z\}$. Thus, the scaling function and the multiwavelet functions have to satisfy the two-scale dilation equations

$$
\Phi(t)=\sqrt{2} \sum_{k} H_{k} \Phi(2 t-k) \text { and } \Psi(t)=\sqrt{2} \sum_{k} G_{k} \Phi(2 t-k),
$$




\section{MULTIWAVELET FUNCTIONS IN IMAGE COMPRESSION}

where $G_{k}$ and $H_{k} \in l^{2}(2)^{N \times N}$ are $N \times N$ matrices of filter coefficients. Moreover, the set of scaling functions $\quad\left\{\phi_{i, j, k}(t): 1 \leq i \leq N, k \in Z\right\} \quad$ with $\phi_{i, j, k}(t)=2^{-j / 2} \phi_{i}\left(t / 2^{j}-k\right)$ is a Riesz basis for $V_{i}$. We also write

$$
\left[\varphi_{1, j, k}(t), \ldots, \varphi_{N, j, k}(t)\right]^{T}=\Phi_{j, k}(t)
$$

In the case of orthonormal multiscaling functions, $\left\{\phi_{i}(t): 1 \leq i \leq N, k \in Z\right\}$ is not just a Riesz basis, but is orthonormal, i.e.,

$$
\langle\Phi(t), \Phi(t-k)\rangle=\int \Phi(t) \Phi^{T}(t-k) d t=I_{N} \delta_{k, 0}, k \in Z
$$

where $I_{N}$ is the $N \times N$ identity matrix. This implies that

$$
\sum_{k} G_{k} G_{2 l+k}^{T}=I_{r} \delta_{0, l}, l \in Z
$$

In scalar wavelets, this means that the sum of squares of low-pass filter coefficients equals unity, and the filter is orthogonal to its even translates.

\section{Linear Legendre Multiwavelets}

A pair of linear Legendre scaling functions $\phi_{1}(x)$ and $\phi_{2}(x)$ on $[0,1]$ are introduced and depicted in Figure 1.

$$
\phi_{1}(x)=1 \text {, and } \phi_{2}(x)=\sqrt{3}(2 x-1) \text {. }
$$




\section{HASHEMIZADEH \& RAHBAR}
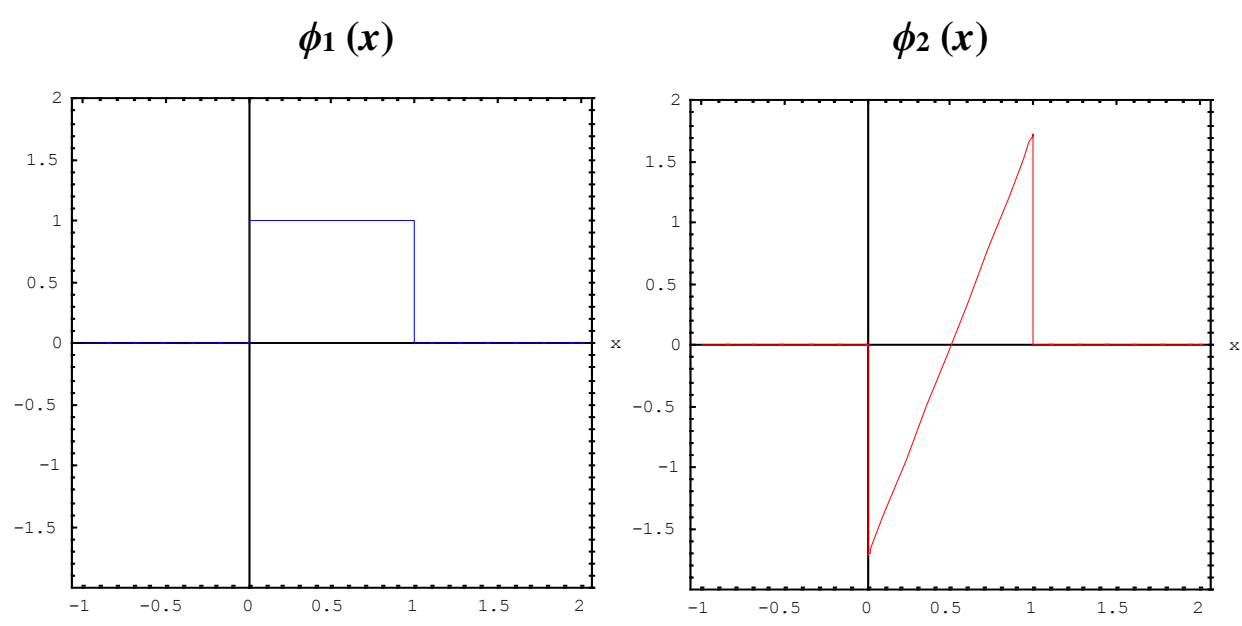

Figure 1. A pair of linear Legendre scaling functions. The left is $\phi_{1}(x)$ and the right is $\phi_{2}(x)$.

The integer translates of $\phi_{1}(x)$ together with the ones of $\phi_{2}(x)$ span a subspace $V_{0}$. Furthermore, the translates of $1 / 2$ scaled version of $\phi_{1}(x)$ and $\phi_{2}(x)$ that span $V_{1} \supset V_{0}$ are given by

$$
\left\{\begin{array}{cc}
\varphi_{1}(2 x-k)=1 & \text { for } \frac{k}{2} \leq x<\frac{k+1}{2} \\
\varphi_{2}(2 x-k)=\sqrt{3}(4 x-2 k-1) & \text { for } \frac{k}{2} \leq x<\frac{k+1}{2}
\end{array}\right.
$$

The two-scale relations for linear Legendre scaling functions should express each of $\phi_{1}(x)$ and $\phi_{2}(x)$ in terms of the four scaling functions $\phi_{i}(2 x)$ and $\phi_{i}(2 x-1)$, $i=1,2$ (Udea \& Lodha, 1995). We propose these relations to be

$$
\left[\begin{array}{l}
\phi_{1}(x) \\
\phi_{2}(x)
\end{array}\right]=\left[\begin{array}{cccc}
1 & 0 & 1 & 0 \\
-\frac{\sqrt{3}}{2} & \frac{1}{2} & \frac{\sqrt{3}}{2} & \frac{1}{2}
\end{array}\right]=\left[\begin{array}{c}
\phi_{1}(2 x) \\
\phi_{2}(2 x) \\
\phi_{1}(2 x-1) \\
\phi_{2}(2 x-1)
\end{array}\right]
$$




\section{MULTIWAVELET FUNCTIONS IN IMAGE COMPRESSION}

Let $\psi_{1}(x)$ and $\psi_{2}(x)$ be wavelets on $[0,1]$ defined based on the linear Legendre scaling functions. By using (1), $\psi_{1}(x)$ and $\psi_{2}(x)$ can be expressed in terms of $\phi_{i}(2 x)$ and $\phi_{i}(2 x-1), i=1,2$. The conditions of being orthonormal and vanishing moments help reduce the two-scale relation for linear Legendre multiwavelets as follows.

$$
\left[\begin{array}{l}
\psi_{1}(x) \\
\psi_{2}(x)
\end{array}\right]=\left[\begin{array}{cccc}
0 & -1 & 0 & 1 \\
\frac{1}{2} & \frac{\sqrt{3}}{2} & -\frac{1}{2} & \frac{\sqrt{3}}{2}
\end{array}\right]\left[\begin{array}{c}
\phi_{1}(2 x) \\
\phi_{2}(2 x) \\
\phi_{1}(2 x-1) \\
\phi_{2}(2 x-1)
\end{array}\right]
$$

Then, the explicit formulae for $\psi_{1}(x)$ and $\psi_{2}(x)$ are

$$
\psi_{1}(x)= \pm\left\{\begin{array}{ll}
-\sqrt{3}(4 x-1), & 0 \leq x<\frac{1}{2} \\
+\sqrt{3}(4 x-3), & \frac{1}{2} \leq x<1
\end{array}, \text { and } \quad \psi_{2}(x)= \pm \begin{cases}6 x-1, & 0 \leq x<\frac{1}{2} \\
6 x-5, & \frac{1}{2} \leq x<1\end{cases}\right.
$$

\section{Decomposition relations}

The matrix equation

$$
\left[\begin{array}{l}
\phi_{1}(x) \\
\phi_{2}(x) \\
\psi_{1}(x) \\
\psi_{2}(x)
\end{array}\right]=\left[\begin{array}{cccc}
1 & 0 & 1 & 0 \\
-\frac{\sqrt{3}}{2} & \frac{1}{2} & \frac{\sqrt{3}}{2} & \frac{1}{2} \\
0 & -1 & 0 & 1 \\
\frac{1}{2} & \frac{\sqrt{3}}{2} & -\frac{1}{2} & \frac{\sqrt{3}}{2}
\end{array}\right]\left[\begin{array}{c}
\phi_{1}(2 x) \\
\phi_{2}(2 x) \\
\phi_{1}(2 x-1) \\
\phi_{2}(2 x-1)
\end{array}\right]
$$

which combines (1) and (2) is called the reconstruction relation. Note that $\phi_{1}(x), \phi_{2}(x) \in V_{0}$ and $\psi_{1}(x), \psi_{2}(x) \in W_{0}$. This four bases are expressed in terms of $\phi_{1}(2 x), \phi_{2}(2 x), \phi_{1}(2 x-1)$ and $\phi_{2}(2 x-1)$ in $V_{1}$ subspace.

The decomposition relation is simply defined as the inverse of the reconstruction relation defined in (3). The square matrix in (3) is orthogonal with constant magnitude. This is because $\left\{\phi_{1}(x), \phi_{2}(x), \psi_{1}(x), \psi_{2}(x)\right\} \quad$ and $\left\{\phi_{1}(2 x), \phi_{2}(2 x), \phi_{1}(2 x-1), \phi_{2}(2 x-1)\right\}$. Thanks to the property $M^{-1}=M^{T}$ of any orthogonal matrix, the decomposition relation would be 


$$
\left[\begin{array}{c}
\varphi_{1}(2 x) \\
\varphi_{2}(2 x) \\
\varphi_{2}(2 x-1) \\
\varphi_{2}(2 x-1)
\end{array}\right]=\frac{1}{2}\left[\begin{array}{cccc}
1 & -\frac{\sqrt{3}}{2} & 0 & \frac{1}{2} \\
0 & \frac{1}{2} & -1 & \frac{\sqrt{3}}{2} \\
1 & \frac{\sqrt{3}}{2} & 0 & -\frac{1}{2} \\
0 & \frac{1}{2} & 1 & \frac{\sqrt{3}}{2}
\end{array}\right]\left[\begin{array}{l}
\varphi_{1}(x) \\
\varphi_{2}(x) \\
\psi_{1}(x) \\
\psi_{2}(x)
\end{array}\right]
$$

\section{Preprocessing and sampling}

Preprocessing aims to convert the given scalar input signal of length $N$ to a sequence of two-dimensional vectors $v_{0, k}$. This is used in the analysis algorithm as shown in Figure 2. Here, $N$ is assumed to be a multiple of a power of 2, and therefore it even. The signal $\left\{X_{k}\right\}$ is taken to be a function $x($.) which is observed at integer time points. If the preprocessing produces $N$ two-dimensional vectors, it is said to be an oversampling scheme. If it produces $N / 2$ two-dimensional vectors, the result is a critical sampling. After the wavelet reconstruction step (synthesis), e.g., inverse DMWT, a postfilter is applied. Evidently, prefiltering, wavelet transform, inverse transform, and postfiltering should exactly recover the input signal.

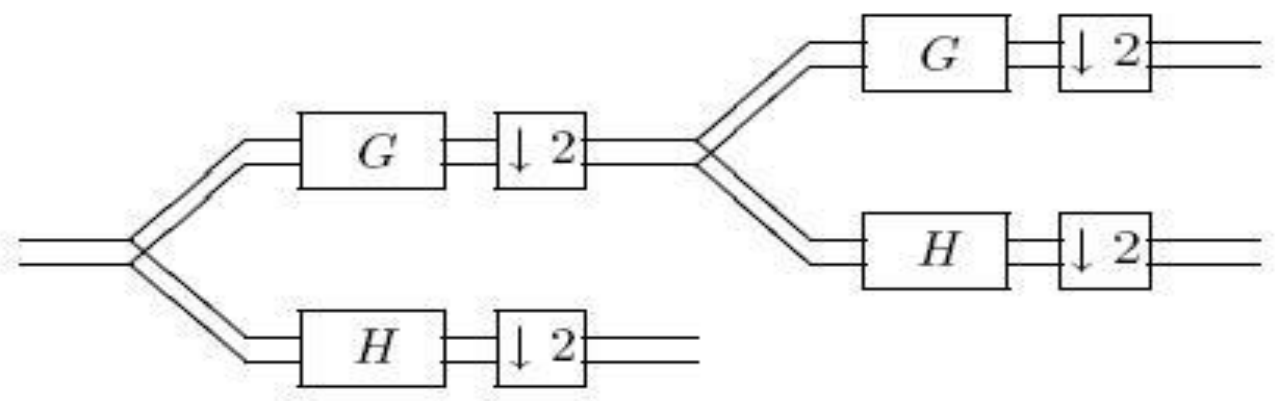

Figure 2. A multiwavelet filter bank which is iterated once. 


\section{MULTIWAVELET FUNCTIONS IN IMAGE COMPRESSION}

\section{Repeated Row Preprocessing: Oversampling Scheme}

The obvious way to get two input rows from a given signal is to repeat the signal. Two identical rows go into the multifilter bank. It then begins oversampling of the data by a factor of two. Although, the usefulness of oversampling has been proven, it requires more calculations than critical sampling. Furthermore, in data compression applications, one intends to remove redundancy, not to increase it. In the case of one-dimensional signals the repeated row scheme is convenient to be implemented (Strela \& Walden, 1998).

\section{Matrix (approximation) Preprocessing: Critical Sampling}

Let $x(t)$ belongs to $V_{0}$ and is generated by the translates of linear Legendre multiscaling functions so that $x(t)=\sum_{k}\left[v_{0, k}^{(0)} \varphi_{1}(t-k)+v_{0, k}^{(1)} \varphi_{2}(t-k)\right]$.

Suppose that the input sequence samples are made at the half-integers, i.e., $X_{2 k}=x(k)$, and $X_{2 k+1}=x\left(k+\frac{1}{2}\right)$.

From Fig. 1 for the linear Legendre multiscaling function, the only nonzero function values at the integers and half-integers are $\phi_{1}(0)=1, \phi_{2}\left(\frac{1}{2}\right)=1$, and $\phi_{2}(0)=-\sqrt{3}$.

Thus,

$$
X_{2 k}=v_{0, k}^{(0)}-\sqrt{3} v_{0, k}^{(1)} \text {, and } X_{2 k+1}=v_{0, k}^{(0)} \text {. }
$$

Hence,

$$
v_{0, k}^{(0)}=X_{2 k+1},
$$

and

$$
v_{0, k}^{(1)}=\frac{1}{\sqrt{3}} X_{2 k+1}-\frac{1}{\sqrt{3}} X_{2 k}
$$

Equations (4) and (5) give the required vector $v_{0, k}$ for $k=0, \ldots, N / 2-1$.We can write it in matrix form as follows. 


$$
\left[\begin{array}{c}
v_{0, k}^{(0)} \\
v_{0, k}^{(1)}
\end{array}\right]=\left[\begin{array}{cc}
0 & 1 \\
-\frac{1}{\sqrt{3}} & \frac{1}{\sqrt{3}}
\end{array}\right]\left[\begin{array}{c}
X_{2 k} \\
X_{2 k+1}
\end{array}\right]
$$

In fact, approximation prefiltering is a special case of matrix prefiltering,

$$
v_{0, k}=\sum_{m=0}^{M} P_{m}\left[\begin{array}{c}
X_{2(m+k)} \\
X_{2(m+k)+1}
\end{array}\right] .
$$

where $P_{0}, P_{1}, \ldots, P_{M}$ are $2 \times 2$ matrices. Thus, for linear Legendre multiwavelets, we have

$$
P_{0}=\left[\begin{array}{cc}
0 & 1 \\
-\frac{1}{\sqrt{3}} & \frac{1}{\sqrt{3}}
\end{array}\right], \text { and } P_{i}=0_{2}, i=1, \ldots, M
$$

where $0_{2}$ is a $2 \times 2$ matrix of zeros.

If prefilters are $N$-dimensional, the matrix preprocessing is represented by $\rho X=V_{0}$ where now $\rho$ is $N \times N, X$ is $N \times 1$, and $V_{0}$ is $N \times 1$. Then, we have

$$
\left[\begin{array}{ccccccc}
P_{0} & P_{1} & \cdots & P_{M} & 0_{2} & \cdots & \cdots \\
0_{2} & P_{0} & P_{1} & \cdots & P_{M} & 0_{2} & \cdots \\
& & & & & & \ddots
\end{array}\right]\left[\begin{array}{c}
X_{0} \\
X_{1} \\
X_{2} \\
X_{3} \\
\vdots
\end{array}\right]=\left[\begin{array}{c}
v_{0,0}^{(0)} \\
v_{0,0}^{(1)} \\
v_{0,1}^{(0)} \\
v_{0,1}^{(1)} \\
\vdots
\end{array}\right]
$$

where $P_{m}$ 's are $2 \times 2$ matrices (Strela, Heller, Strang, Topiwala, \& Heil, 1999; Strela, 1996; Kim \& Li, 2003).

\section{Image Compression Using Legendre Wavelets}

The notions stated above were applied to compress a given image. In doing so, critical sampling was deployed on a well-known photo of Barbara. Initially 


\section{MULTIWAVELET FUNCTIONS IN IMAGE COMPRESSION}

preprocess all the rows (see Strela \& Walden, 1998), which results in the picture shown in Figure 3.

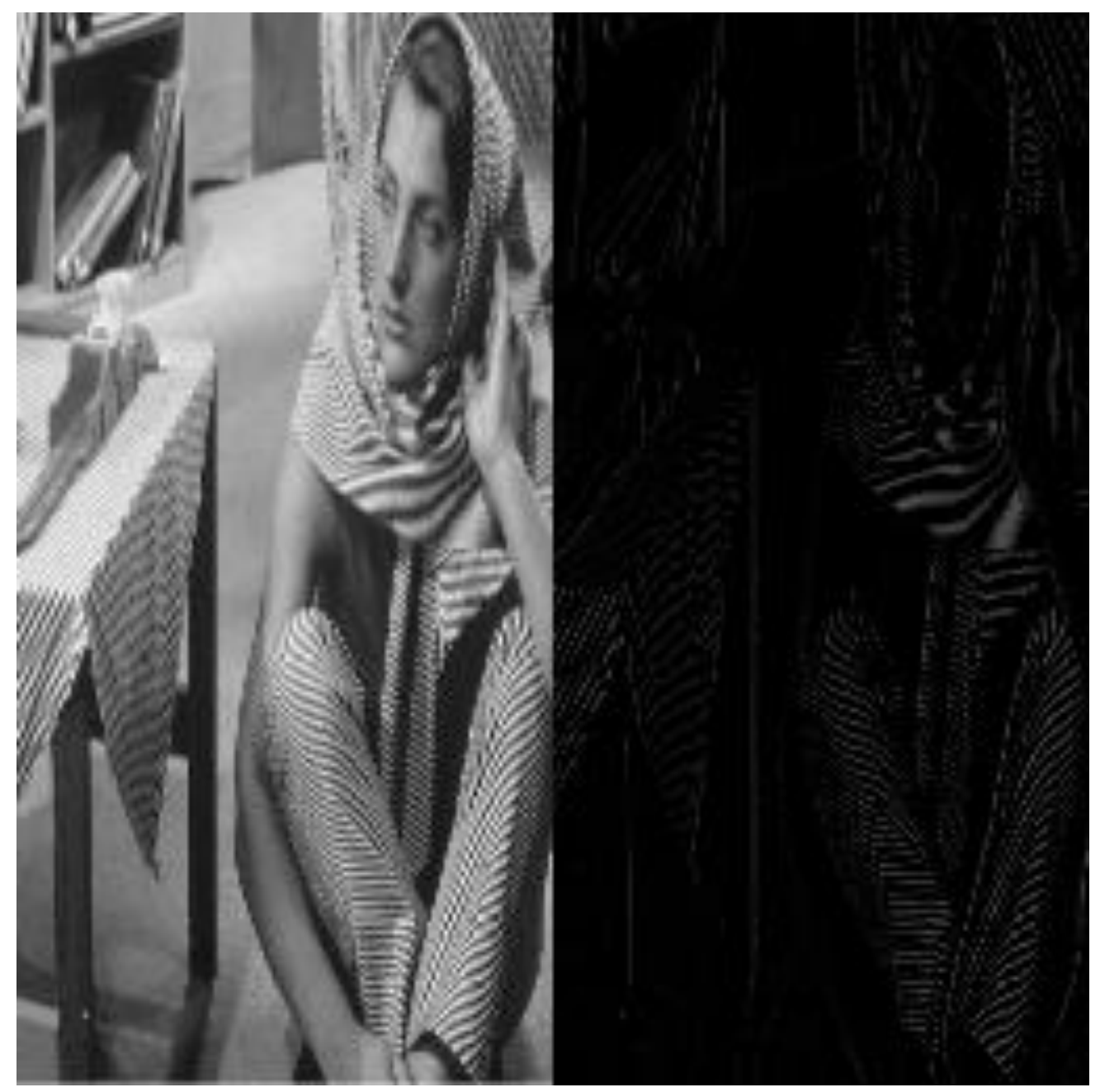

Figure 3. The result of row preprocessing on Barbara's photo.

Then, preprocess all columns. The amount of data remains unchanged, since we use critical sampling. 


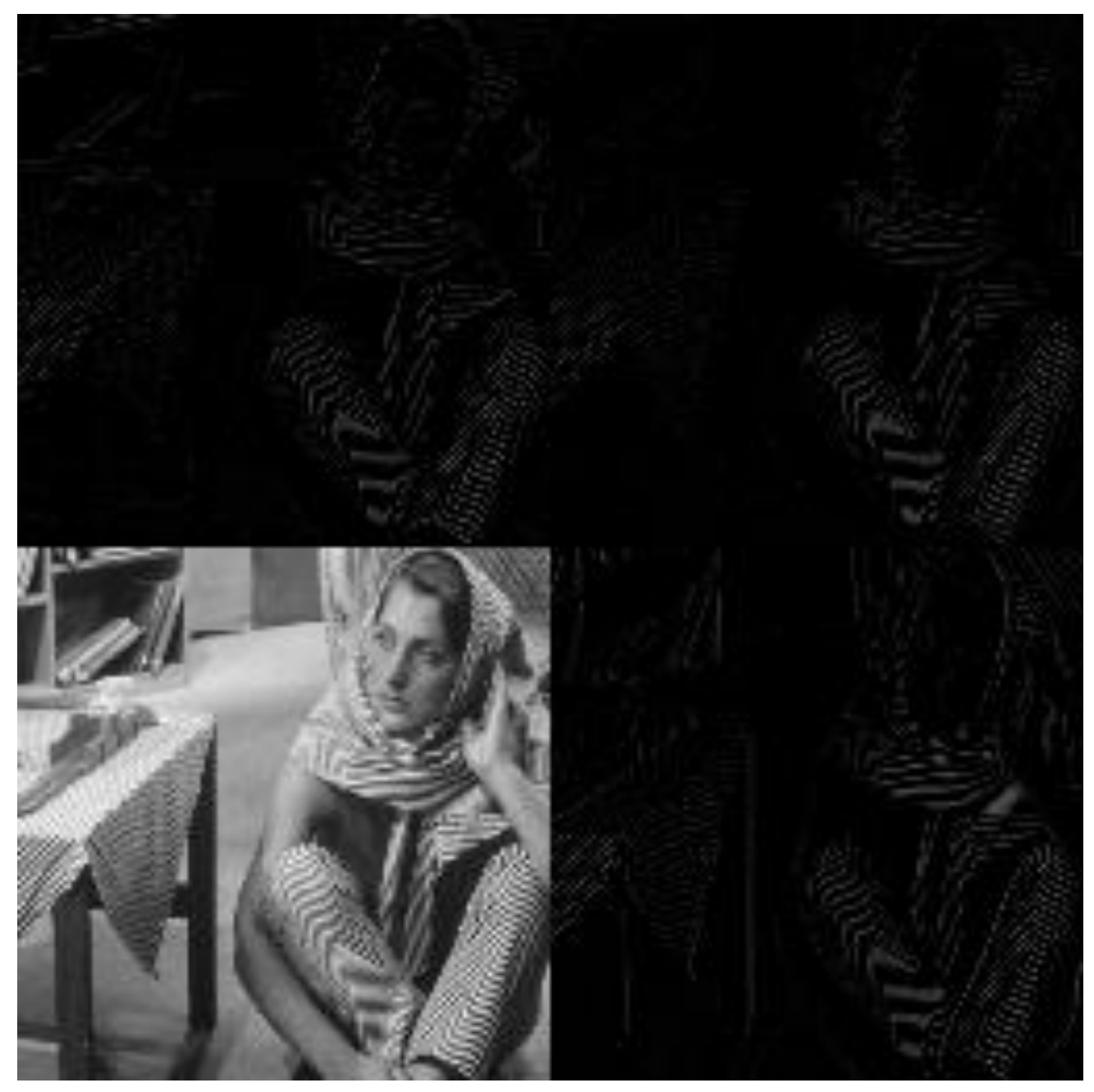

Figure 4. Result of column preprocessing on the photo in Figure 3.

At the next step, perform the 2D-wavelet cascade. For linear Legendre multiwavelets, the multiwavelet filter bank is obtained as follow. From (3), we have

$$
H_{1}=\left[\begin{array}{cc}
1 & 0 \\
-\frac{\sqrt{3}}{2} & \frac{1}{2}
\end{array}\right], H_{2}=\left[\begin{array}{cc}
1 & 0 \\
\frac{\sqrt{3}}{2} & \frac{1}{2}
\end{array}\right], G_{1}=\left[\begin{array}{cc}
0 & -1 \\
\frac{1}{2} & \frac{\sqrt{3}}{2}
\end{array}\right], \text { and } G_{2}=\left[\begin{array}{cc}
0 & 1 \\
-\frac{1}{2} & \frac{\sqrt{3}}{2}
\end{array}\right]
$$




\section{MULTIWAVELET FUNCTIONS IN IMAGE COMPRESSION}

where $H_{1}, H_{2}, G_{1}$, and $G_{2}$ are matrix filters. Using the ideas of matrix block multiplication (Van Fleet, 2000), the above filters can be combined into the matrix below.

$$
\left[\begin{array}{llllllllll}
\ddots & \ddots & \ddots & & & & & & & \\
& 0_{2} & H_{1} & H_{2} & 0_{2} & 0_{2} & 0_{2} & 0_{2} & 0_{2} & \\
\cdots & 0_{2} & 0_{2} & 0_{2} & H_{1} & H_{2} & 0_{2} & 0_{2} & 0_{2} & \cdots \\
& 0_{2} & 0_{2} & 0_{2} & 0_{2} & 0_{2} & H_{1} & H_{2} & 0_{2} & \\
\hline & 0_{2} & G_{1} & G_{2} & 0_{2} & 0_{2} & 0_{2} & 0_{2} & 0_{2} & \\
\cdots & 0_{2} & 0_{2} & 0_{2} & G_{1} & G_{2} & 0_{2} & 0_{2} & 0_{2} & \cdots \\
& 0_{2} & 0_{2} & 0_{2} & 0_{2} & 0_{2} & G_{1} & G_{2} & 0_{2} & \\
& & & & & & & \ddots & \ddots & \ddots
\end{array}\right],
$$

where $0_{2}$ is a $2 \times 2$ zero matrix. The two-channel matrix filter bank operates on two input data streams, filters them into four output data streams, and then, downsamples each of the results by a factor of two. After one step of the 2D multiwavelet cascade algorithm for linear Legendre with approximation preprocessing, the photo of Figure 5 appears. 


\section{HASHEMIZADEH \& RAHBAR}

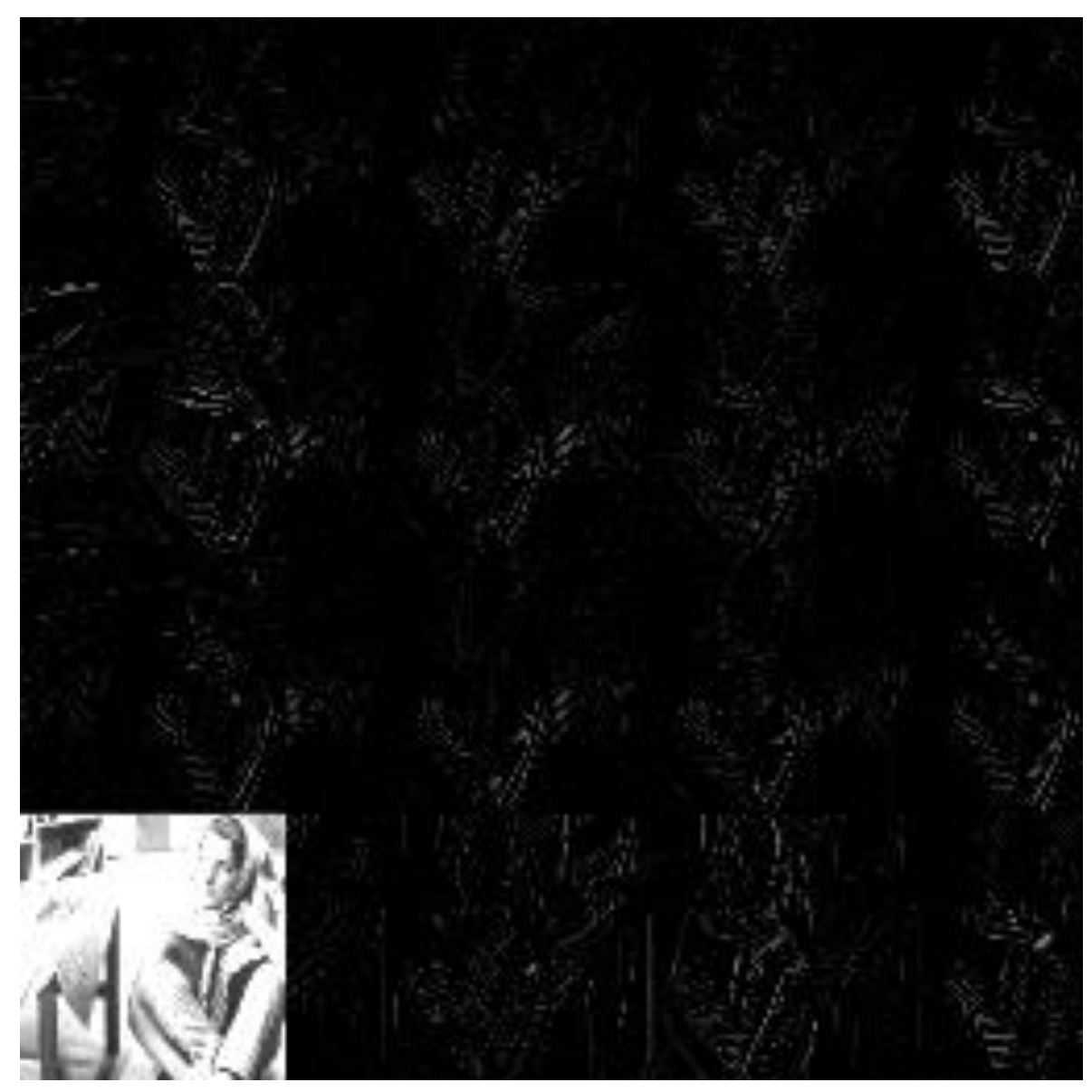

Figure 5. The result of one-step 2D multiwavelet cascade algorithm with linear Legendre wavelets and approximation preprocessing.

The pattern of image subbands is shown in Figure 6 (Sudhakar \& Jayaraman, 2005). By comparing Figure 5 with Figure 6, it is seen that almost all information is in the block of $G_{1} G_{1}$. This reflects the fact that, for linear Legendre, one scaling function is symmetric while the other is anti-symmetric. 


\section{MULTIWAVELET FUNCTIONS IN IMAGE COMPRESSION}

\begin{tabular}{|l|l|l|l|}
\hline$H_{2} G_{1}$ & $H_{2} G_{2}$ & $H_{2} H_{1}$ & $H_{2} H_{2}$ \\
\hline$H_{1} G_{1}$ & $H_{1} G_{2}$ & $H_{1} H_{1}$ & $H_{1} H_{2}$ \\
\hline$G_{2} G_{1}$ & $G_{2} G_{2}$ & $G_{2} H_{1}$ & $G_{2} H_{2}$ \\
\hline$G_{1} G_{1}$ & $G_{1} G_{2}$ & $G_{1} H_{1}$ & $G_{1} H_{2}$ \\
\hline
\end{tabular}

Figure 6. The pattern of image subbands.

Figure 7 compares the cumulative energy in the original image with the one resulted from one-step Legendre multiwavelet transform.

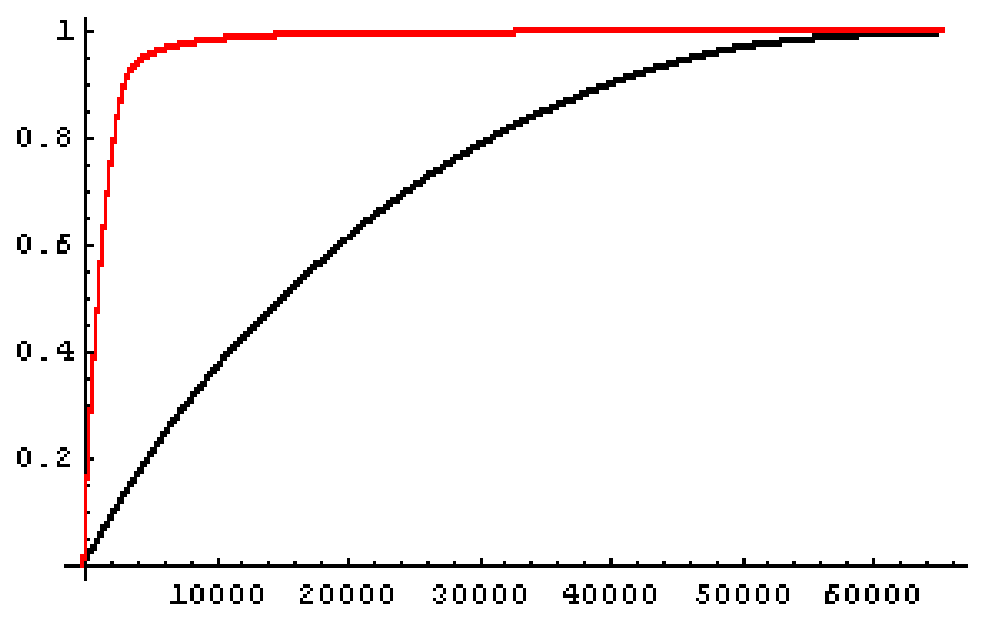

Figure 7. The cumulative energy of the original image and the one produced by the onestep Legendre multiwavelet transform.

The next step is to quantize the components of the Legendre multiwavelet transform. Any values of the transform that are small in modulus will be 


\section{HASHEMIZADEH \& RAHBAR}

converted to 0 . The modified transform is then be converted to Huffman codes and the resulting bit stream is encoded. The produced file is markedly smaller than the original one.

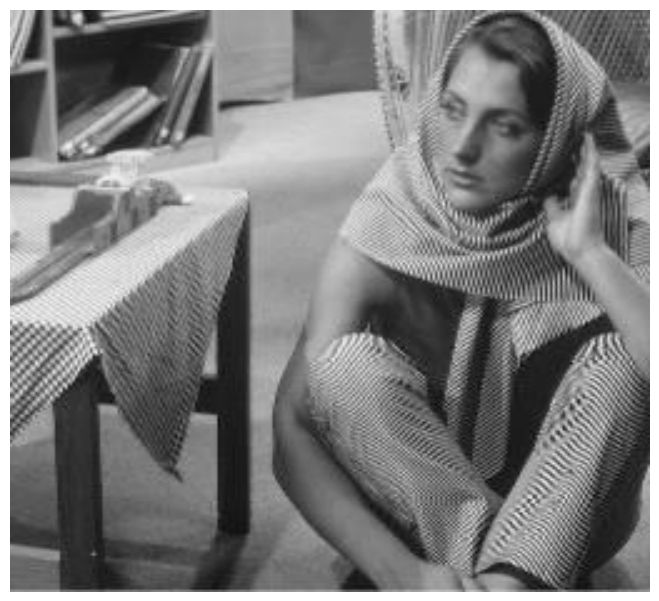

Original Barbara image

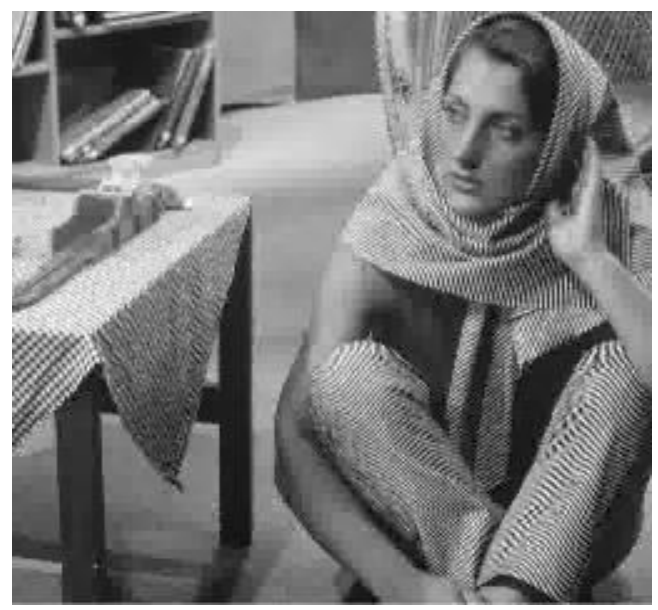

Compression 32:1, PSNR 30.31

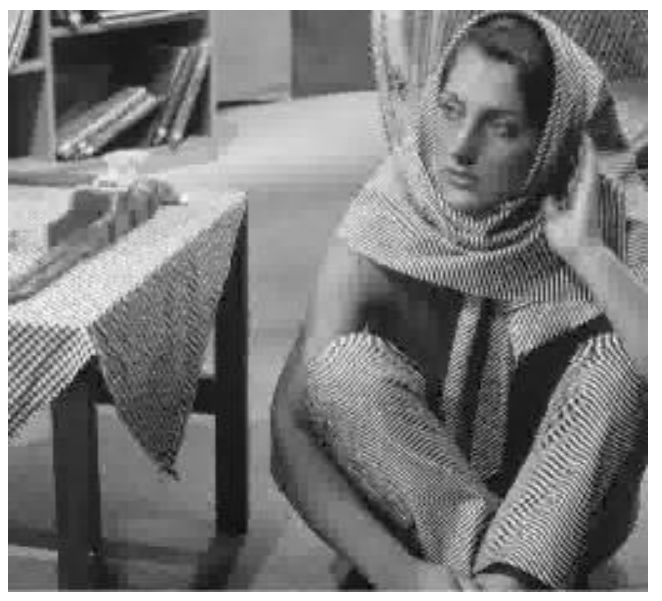

Compression 64:1, PSNR 29.1

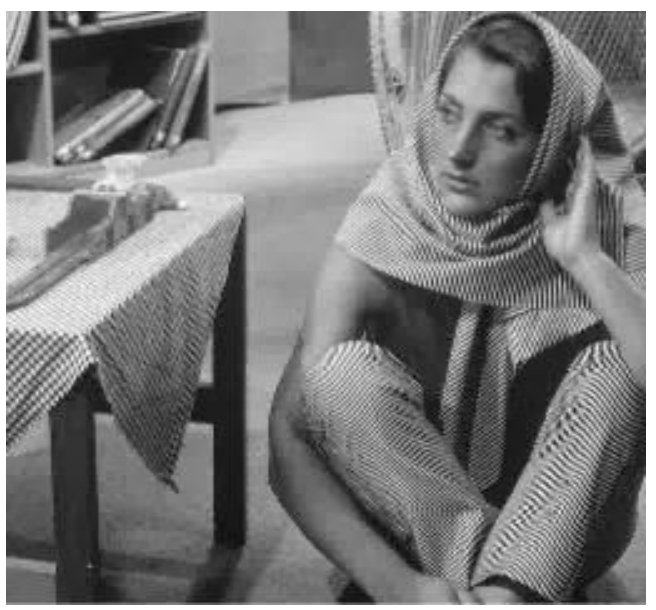

Compression 16:1, PSNR 34.8

Figure 8. Some implementations of linear Legendre multiwavelet compression on Barbara's image. 


\section{MULTIWAVELET FUNCTIONS IN IMAGE COMPRESSION}

\section{Conclusion}

The technique of using Legendre multiwavelets in image compression, as deployed in this paper, shows great advantages compared to the wavelet-based and even other multiwavelet-based methods. This is due to the fact that in a linear Legendre multiwavelet one scaling function is symmetric while the other one is anti-symmetric. In this way, it concentrates well on the image and excludes a tiny amount of details. Linear Legendre multiwavelets were used, but, the higher order Legendre multiwavelets can be employed (Rahbar, 2004).

\section{References}

Aboufadel, E. \& Schilicker, S. (1999). Discovering wavelets. New York, NY: Wiley.

Barbara [Digital Image]. Retrieved from https://homepages.cae.wisc.edu/ ece533/images/barbara.bmp

Burrus, C. S., Gopinath, R. A., \& Guo, H. (1998). Introduction to wavelets and wavelet transforms: A primer. Upper Saddle River, NJ: Prentice Hall.

Daubechies, I. (1992). Ten lectures on wavelets. Philadelphia, PA: SIAM.

Kim, W., \& Li, C.-C. (2003). On preconditioning multiwavelet system for image compression. International Journal of Wavelets, Multiresolution and Information Processing, 1(1), 51-74. doi: 10.1142/S0219691303000049

Martin, M. B. \& Bell, A. E. (2001). New image compression techniques using multiwavelets and multiwavelet packets. IEEE Transactions on Image Processing, 10(4), 500-510. doi: 10.1109/83.913585

Rahbar, S. (2004). Solving Fredholm Integral Equation Using Legendre Wavelet Functions. WSEAS Transactions on Mathematics, 3(3), 591-594. doi: 10.1.1.552.2048

Strela, V. (1996). Multiwavelets: Theory and applications (Doctoral dissertation). Massachusetts Institute of Technology, Cambridge, MA.

Strela, V., Heller, P. N., Strang, G., Topiwala, P., \& Heil, C. (1999).The application of multiwavelet filterbanks to image processing. IEEE Transactions on Image Processing, 8(4), 548-563. doi: 10.1109/83.753742

Strela, V. \& Walden, A. (1998). Signal and image denoising via wavelet thresholding: Orthogonal and biorthogonal, scalar and multiple wavelet 


\section{HASHEMIZADEH \& RAHBAR}

transforms (Technical Report TR-98-01). London: Imperial College Statistics Section.

Sudhakar, R \& Jayaraman, S. (2005). Fingerprint compression using multiwavelets. International Journal of Signal Processing, 2(2), 78-87.

Udea, M \& Lodha, S. (1995). Wavelets: An elementary introduction and examples (Technical Report). Santa Cruz, CA: University of California at Santa Cruz.

Van Fleet. P. J. (2000). An elementary introduction to wavelets and their applications. Unpublished manuscript. 